\section{MATURATIONAL CHANGES OF AMPLITUDE INTEGRATED EEG IN PRETERM INFANTS}

\author{
E. Griesmaier ${ }^{1}$, V. Neubauer ${ }^{1}$, C. Mulser ${ }^{1}$, \\ U. Kiechl-Kohlendorfer ${ }^{1}$, M. Keller ${ }^{2}$
}

${ }^{1}$ Department of Pediatrics IV, Medical University of Innsbruck, Innsbruck, Austria, ${ }^{2}$ Department of Pediatrics I, Neonatology, University Hospital of Essen, Essen, Germany

Background and aims: Improvement of neonatal care during the last decades caused an increase in the number of surviving preterm born infants. In particular very low birth weight infants (VLBW, $<1000 \mathrm{~g}$ ) have a high risk of brain injury and developmental deficits. Beside morphological diagnostics (ultrasonography, magnet resonance imaging, a.o.m.), the amplitude integrated EEG (aEEG) preserves the possibility to monitor the functional status of the preterm brain and to observe maturational changes during long-term development. Analysis of physiological changes is the basis for interpreting pathological cerebral function. We aimed to characterize developmental changes in aEEG and to compare published methods with respect to their correlation with gestational age.

Methods: Infants born < 32 weeks gestational age without structural brain pathologies were included to the study. aEEG recordings were obtained weekly up to 28 days after birth. Minimum and maximum amplitude, background pattern, sleep wake cycling, number of burst per hour were examined and correlated to the Burdjalov score (Burdjalov, Pediatrics 2003; Hellström-Westas, NeoReviews 2006).

Results: 19 babies were included into the study. Minimal amplitude (MA), time point of appearance of sleep wake cycling (SWC) and duration of continuous background (DCP) pattern as well as the Burdjalov score have shown a statistically significant correlation with postmenstrual age.

Conclusion: MA, background pattern and occurence of SWC can be used for the evaluation of brain maturation in preterm infants. We hypothesize that a delay or disturbance of brain developmental is reflected in deviation of the above described aEEG changes. Further analysis are ongoing.

\section{CEREBRAL VENOUS SINUS THROMBOSIS: PREDISPOSING FACTORS AND OUTCOMES}

\author{
N.F. Ismail ${ }^{1}$, C. John², R. Clarke ${ }^{3}$ \\ ${ }^{1}$ Neonatal Unit, Liverpool Women's NHS \\ Foundation Trust, Liverpool, ${ }^{2}$ Paediatrics, ST \\ Helens and Knowsley Hospitals, Preston, ${ }^{3}$ Ear, \\ Nose and Throat, Royal Liverpool Children's \\ Hospital, Liverpool, UK
}

Introduction: Cerebral venous sinus thrombosis (CVST) is difficult to diagnose. It can be lifethreatening and can cause long term neurological deficit.

Aim: Determine the underlying cause and outcome.

Methods: Retrospective analysis of casenotes of patients diagnosed with CVST between 1997-2009 in a tertiary Children's hospital.

Results: Seventeen patients were identified from clinical coding (10 male, 7 female). 6/17 patients had infection of the ears/mastoid as the main predisposing factor, $3 / 17$ had leukaemia and $2 / 17$ had central lines in situ. 2/17 were felt to be secondary to non accidental head injury. One 14 year old girl was on the contraceptive pill. One patient had Transposition of great vessels. Only one patient was clinically significantly dehydrated. All patients had MR venography done. There was no family history of thrombophilia.Median age of presentation was 5.5 years (range 2 days- 17 years). $6 / 17$ had vomiting as a presenting feature.11/17 had abnormal neurology. 6/17 had papilloedema. Only one patient was hypertensive. $3 / 17$ had positive bacterial cultures (2 blood, 1 pus). Thrombophilia screens were abnormal in 5/17 (2 heterozygote for factor $V$ wild type). 7/17 had complete recovery. $3 / 17$ had residual neurological deficit, $2 / 17$ suffered persistent headache, 1 had middle ear effusion, 2 died and no follow up data on 2 patients.

Conclusion: The commonest predisposing factors for CVST are sinus/mastoid infections. MRI/MRV remains the gold standard for diagnosis. There needs to be a high index of suspicion and a low threshold to perform MRI scans in suspected CVST as it can be life-threatening and can cause long term neurological deficit. 